Journal of Computer Science 7 (5): 671-677, 2011

ISSN 1549-3636

(C) 2011 Science Publications

\title{
Design of Synthetic Optimizing Neuro Fuzzy Temperature Controller for Dual Screw Profile Plastic Extruder Using Labview
}

\author{
${ }^{1}$ Ravi Samikannu and ${ }^{2}$ Balakrishnan Anantharaman Poonamallie \\ ${ }^{1}$ Department of Electrical and Electronics Engineering, Nandha Engineering College, Erode, India \\ ${ }^{2}$ Dean (Academic and Research), KCG College of Technology, Chennai, Tamilnadu, India
}

\begin{abstract}
Problem statement: The temperature control in plastic extrusion machine is an important factor to produce high quality plastic products. The first order temperature control system in plastic extrusion comprises of coupling effects, long delay time and large time constants. Controlling temperature is very difficult as the process is multistage process and the system coupled with each other. In order to conquer this problem the system is premeditated with neuro fuzzy controller using LabVIEW. Approach: The existing technique involved is conventional PID controller, Neural controller, mamdani type Fuzzy Logic Controller and the proposed method is neuro fuzzy controller. Results: Manifest feature of the proposed method is smoothing of undesired control signal of conventional PID, neural controller and mamdani type FLC controller. The software incorporated the LabVIEW graphical programming language and MATLAB toolbox were used to design temperature control in plastic extrusion system. Hence neuro fuzzy controller is most powerful approach to retrieve the adaptiveness in the case of nonlinear system. Conclusion: The tuning of the controller was synchronized with the controlled variable and allowing the process at its desired operating condition. The results indicated that the use of proposed controller improve the process in terms of time domain specification, set point tracking and also reject disturbances with optimum stability.
\end{abstract}

Key words: PID controller, neural controller, plastic extrusion, fuzzy controller, extrusion system, neuro fuzzy controller, synthetic optimizing, polymer material, Nichols tuning rules, PVC extrusion plant, LabVIEW program

\section{INTRODUCTION}

Plastic extrusion is a well known process and widely used in polymerization industry. The extrusion consists of large barrel divided into three temperature zone namely barrel, adapter and die zone respectively. The temperature zone uses more number of heaters in order to provide different temperature ranges. The polymer is fed in to the hooper in solid pellet forms and it is pushed forward by a powerful screw. While passing through the various temperature zones the raw polymer is gradually heated. The heat produced by the heaters in the barrel, together with the heat released from the friction between the raw polymer and the surfaces of the barrel and screw, causes the melting of feed polymer. The melted polymer material is pushed forward by a powerful screw and passes through the molding mechanism from the die. The quality of extrudates depend on uniform temperature distribution, magnitude of the temperature in the barrel, back pressure, physical property of raw material and homogeneity of physical mixing, The temperature section of PVC extrusion plant is shown in Fig. 1.The barrel zone section consists of four sections with eight heaters in different ranges. Adapter section is placed between die zone and barrel zone having a single heater. The die zone consists of six stages and each consists of individual heater. High efficient plastic extrudates can be obtained only when temperature in all the zones is precisely controlled. The implementation of PID controllers retunes their three term parameters so as to ensure that the dynamic behavior of extruder performance is satisfactory along with the specific heat, thermal conductivity and ambient temperature which vary with time. However PID controller perform well only at a particular operating range and it is necessary to retune the PID controller if operating range is changed. The PID controllers do not provide contented results for nonlinear and dead time process (Chamsai et al., 2010).

Corresponding Author: Ravi Samikannu, Department of Electrical and Electronics Engineering,

Nandha Engineering College, Erode, India 


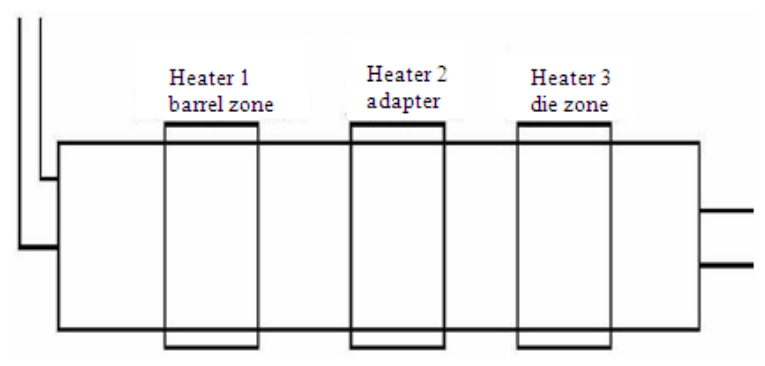

Fig. 1: Temperature section of PVC plant

The Artificial Neural intelligent control scheme can be trained to perform as a controller by learning inverse model dynamics of the plant or as a predictor by identifying the forward model and able to control temperature of the system. When there can be significant uncertainties and changes in the plant dynamics, intelligent adaptive control lends itself as a powerful control strategy and dynamic back propagation learning algorithm is widely used for tuning of the corresponding neural network structures. The convergence speed of this learning method is very slow especially when the search space is complex. This leads to poor transient responses because of the slow error convergence and may be undesirable for tasks requiring precision. So these problems make to design the fuzzy controller to control the temperature. Fuzzy controller performances are less sensitive to parametric variations. The fuzzy controller can be designed without knowing the mathematical model of system. Fuzzy logic controllers have been reported successful for a number of complex and nonlinear processes. The proposed neuro fuzzy controller has the integral advantages of neural and fuzzy approaches and they are used for intelligent decision making systems. Neural networks and fuzzy logic represent two distinct methodologies to deal with uncertainty. Neural networks can model complex non linear relationships and are quietably suited for classification phenomenon of predetermined classes. Neural networks and fuzzy logic are different technologies which can be used to accomplish the specification of mathematical relationships. Among numerous variables in a complex dynamic process, these perform mappings with some degree of imprecision in different ways used to control non linear systems.

Temperature system in plastic extrusion model: Step response method is based on transient response tests (Oreste, 2009). Many industrial processes have step responses of the system in which the step response is monotonous after an initial time. $\mathrm{G}(\mathrm{s})$ is the transfer function of the plant. The transfer function of plastic extrusion pipeline described is given in (1) and the plastic extrusion model uses the parameters $\mathrm{K}=0.92$, $\mathrm{T}=144 \mathrm{~S}, \tau=10 \mathrm{Seconds}$ and the temperature generally varies from $150-200{ }^{\circ} \mathrm{C}$ :

$$
G(s)=\frac{0.92}{1+144 s} e^{-10 \tau}
$$

Conventional controller: Over the years control of process system plants in the industry is customarily done by experts through the conventional PID control techniques. $90 \%$ of the controllers in industry use PID because of its simplicity, low cost design and robust performance in a wide range of operating conditions. The PID control is designed to ensure the specifying desired nominal operating point for temperature control of plastic extrusion model and regulating it, so that it stays closer to the nominal operating point in the case of sudden disturbances, set point variations and noise. Artificial neural network is data processing systems consisting of large number of simple highly inter connected processing elements in architecture inspired by the structure of the cerebral cortex of the brain. The neural networks exhibit mapping capabilities so that they can map input patterns to their associated output patterns. The dynamic back propagation algorithm is subsequently applied to achieve fine adjusting of the network weights (Sharifi et al., 2009). At every time the solution with the best fitness function is used for current representation of the neural network weights and biases. Neural networks can approximate any nonlinear continuous function to an arbitrary accuracy. It can be seen that the net has a layered structure and consists of input units, output units, hidden units and context units. External inputs are received by the input units and propagated forward to the hidden units. The outputs of the hidden units are then sent to the output units to generate the network outputs. In meantime, the outputs of the hidden units are sent to the context units which can be considered to be memory units with one step time delay. The outputs of the context units are also part of the inputs to the hidden units. For a SISO system, only one input line and one output line are required for the Elman network that is simpler than the tapped delay line method commonly adopted when feed forward nets are employed for system identification. The approach begins with judicious selection of neural network models which are trained off line to learn the inverse and forward models of each channel of the 
furnace. The training patterns are obtained from the input output behavioral characteristic of each channel of the process (Malar and Thyagarajan, 2009). Fuzzy logic control constitutes one of the fast growing areas of control technology development and has better prospects. Fuzzy logic is more effective in feedback control system and easier to implement. Fuzzy controller consists of a fuzzifier, rule base and an inference engine, defuzzifier (Ahmad and Mohamed, 2009). The numerical input values of the fuzzifier are converted into fuzzy values, along with the rule base which are fed into the inference engine which produces control value. In fuzzy rule base various rules are fostered according to their respective problem requirements. The control values are not in usable form henceforth are converted to numerical output values using the defuzzifier. Fuzzy control system divides into the single variable fuzzy control system and the multivariable fuzzy control system. It has two input variables, Error $\mathrm{E}$, change in error $\mathrm{CE}$ and one output U. For computation to be relatively simple the triangular shape membership function is used. The computational structure of FLC scheme is composed of the steps rule base and membership function. The fuzzy control rules were formulated in the IF-THEN rules form. The rule base stores the rules governing the input and output relationship of proposed control logic (Pratumsuwan et al., 2010). Fuzzy logic provides an inference morphology that enables approximate human reasoning capabilities to be applied to knowledge based systems. The inference result of each rule consists of two parts, the weighing factor $\mathrm{W}_{\mathrm{i}}$ of the individual rule and the degree of change of temperature $\mathrm{C}$ according to the rule shown in (2):

$$
\mathrm{Z}_{\mathrm{i}}=\min \left\{\mu_{\mathrm{e}}\left(\mathrm{e}_{\mathrm{o}}\right), \mu_{\mathrm{ce}}\left(\mathrm{Ce}_{\mathrm{o}}\right)\right\}, \mathrm{C}_{\mathrm{i}}=\mathrm{w}_{\mathrm{i}} \mathrm{C}_{\mathrm{i}}
$$

where, $\mathrm{Z}_{\mathrm{i}}$ denotes the change in control signal inferred by the $i^{\text {th }}$ rule and $\mathrm{C}$ is noted from the rule table which shows the mapping from the product space of e and ce to $\mathrm{C}_{\mathrm{i}}$ (Liang et al., 2010). The defuzzification method converts the fuzzy output from the inference mechanism to a real word crisp value which is in (3):

$$
\mathrm{Z}=\delta \mathrm{u}_{\mathrm{k}}=\frac{\sum_{i=1}^{\mathrm{N}} \mathrm{z}_{\mathrm{i}}}{\sum_{\mathrm{i}=1}^{\mathrm{N}} \mathrm{w}_{\mathrm{i}}}
$$

Neuro fuzzy controller model: Fuzzy logic and neural networks are natural complementary tools in building intelligent systems. Neural networks are computational structures that perform well when dealing with raw data, fuzzy logic deals with reasoning, using linguistic known as fuzzy rule based systems containing a number of fuzzy IF-THEN rules. Neuro information acquired from domain experts. Fuzzy systems lack the ability to learn and cannot adjust themselves to a new environment. Neural networks can learn, they are opaque to the user (Magaji and Mustafa, 2009). It offers a promising approach to building intelligent systems. Integrated systems can combine the parallel computation and learning abilities of neural networks, with the human like knowledge representation and explanation abilities of fuzzy systems. Fuzzy inference systems are also fuzzy controller is used in the form of sugeno model to integrate the best features of fuzzy systems and neural networks. Neuro fuzzy controller is also used in representation of prior knowledge into a set of constraints to reduce optimization search space obtained from fuzzy and adaptation of back propagation to structured network through neural network (Mjalli et al., 2010).

LabVIEW model of plastic extrusion system: LabVIEW is a programming environment and the programs can be created using graphical notation (connecting functional nodes via wires through which data flows). A LabVIEW program consists of one or more virtual instruments. Virtual instruments are called such because their appearance and operation often imitate actual physical instruments (Iranian and Zabihinejad, 2011). A Virtual instrument created by LabVIEW has two main parts, front panel and block diagram. The front panel is the interactive user interface of a VI, so named because it simulates the front panel of a physical instrument. The front panel shows user inputs, indicators and outputs. The block diagram is the actual executable program. The components of a block diagram are lower level virtual instruments, built in functions, constants and program execution control structures. Front panel objects have corresponding terminals on the block diagram so data can pass from the user to the program and back to the user (Yuwono, 2009).

\section{MATERIALS AND METHODS}

Figure 2 shows the simulink model of block of PID controller. In this case the PID controller optimal setting values obtained through Zeigler Nichol s training rule. The proportional controller stabilizes the gain but produces a steady state error. The integral controller reduces and eliminates the steady state error. The derivative error reduces the rate of change of error. 


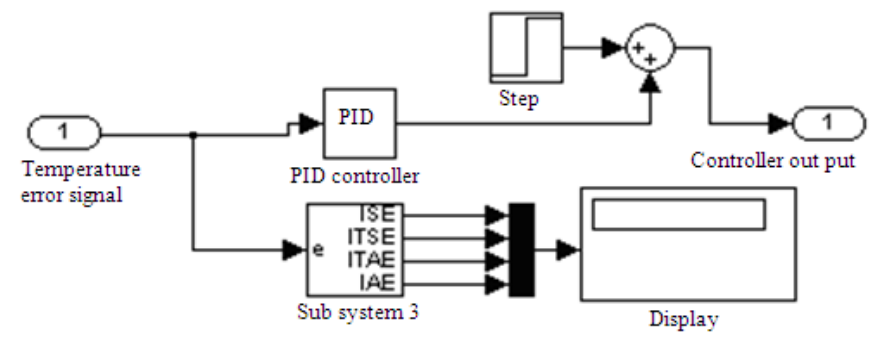

Fig. 2: Simulink model of PID controller

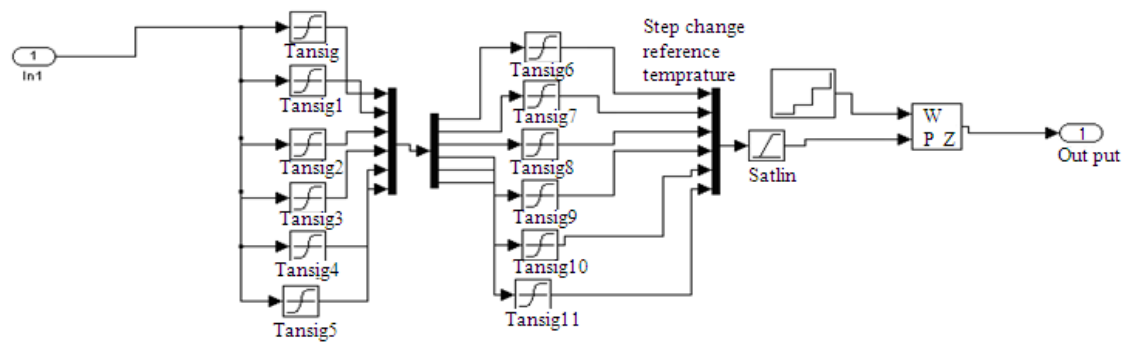

Fig.3: Simulink model of neural network controller

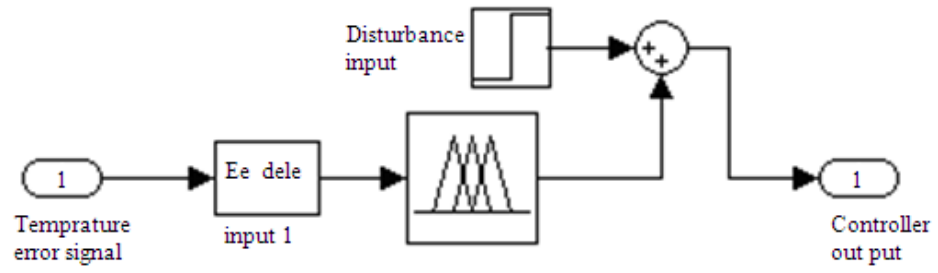

Fig.4: Simulink model of Fuzzy controller

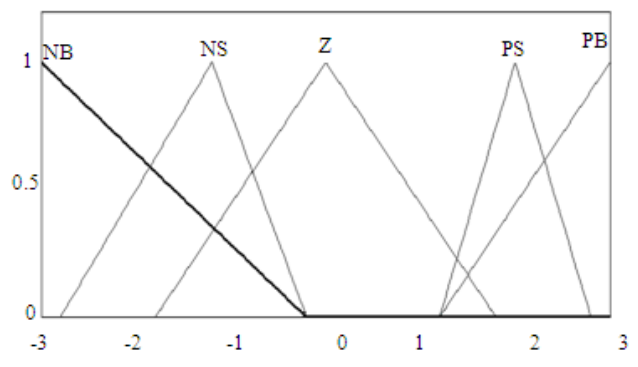

Fig.5: Fuzzy controller output variable u

The neural network simulation model is shown in Fig. 3. Only the feed forward connections are modifiable. The recurrent connections from hidden units to context units are not modifiable and usually their strengths are fixed at 1 . At the beginning of training, the outputs of the context units are made equal to 0 (for hyperbolic tangent activation functions) or 0.5 (for sigmoid activation functions). The weights of the feed forward connections can be modified using BP algorithm. It is set to be the same for all self connections and is not modified by the training algorithm. Since a dynamic system represents a relation between its present output and its past inputs and outputs, the introduction of self feedback in the context units increases the possibility of the Elman net to model high order systems. The implementation of network structure to system identification can give quite satisfactory results that are comparable to or even in some cases better than those achieved by using feed forward networks. The simulink model block of Fuzzy control is shown in Fig. 4. The proposed control algorithm summarizes 25 rules for fuzzy logic. Each universe of discourse is divided into five fuzzy subsystems namely NB, NS, Z, PS and PB. The output variable of fuzzy set $u$ is shown in Fig. 5. 


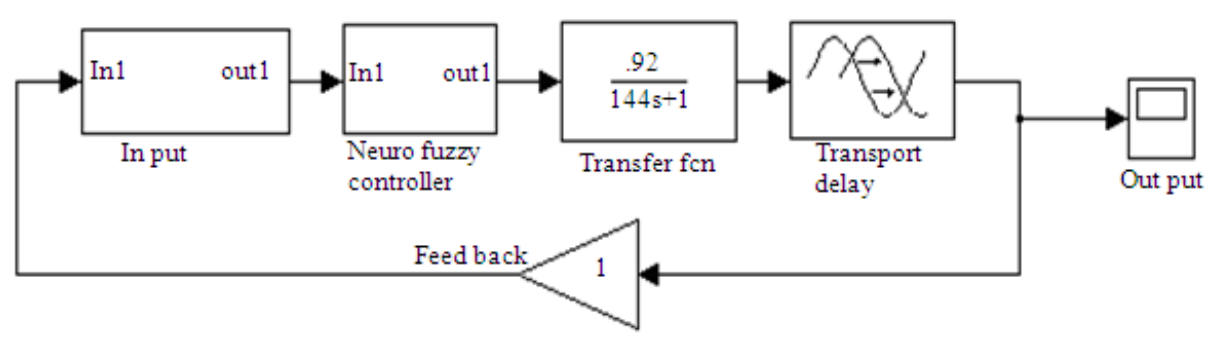

Fig. 6: SIMULINK model of neuro fuzzy controller

The inference mechanism used for evaluating linguistic descriptions (Liang et al., 2010). The fuzzy control rules have been described using linguistic variables for example if error e is NS and the increasing change in error ce is PB then the output is PS to control the temperature rise. The simulink model block of neuro fuzzy controller is shown in Fig. 6. The proposed algorithm summarizes 25 rules. In a neuro fuzzy controller training algorithm each epoch is composed of a forward pass and backward pass. In the forward pass a training set of input patterns is presented to the neuro fuzzy controller, neurons outputs are calculated on the layer by layer basis and the rules consequent parameters are identified by the least squares estimator. The Neuro fuzzy system consists of the components of a conventional fuzzy system. Neuro fuzzy controller uses Takagi Sugeno type FLC.

\section{RESULTS}

The execution of simulation was done using the LabVIEW graphical programming and matlab toolbox. The system is a multistage and coupled system so four set points is taken for the system. Four set point temperatures $70,100,150$ and $200{ }^{\circ} \mathrm{C}$ changes at different times are used in 14000 seconds. Experimental results using LabVIEW for simulation and uses of the first order function. The results of PID Controller for temperature set points is shown in Fig. 7 and it is observed that the performance of the system with PID controller is almost oscillating and takes more time to settle with reference temperature compared with other types of controller. FLC is designed using 5 linguistic levels and 25 rules. The neural network controller output is shown in Fig. 8 which eliminates the peak overshoot but take more time to settle with reference value. FLC simulation output Fig. 9 gives better results compared to PID and neural controller and gives a quick settle with reference temperature with less oscillatory response. The integrated neuro fuzzy controller output with Takagi sugeno fuzzy model is shown in Fig. 10 which eliminates the oscillatory output of different temperature set points and identifies the process variations quickly and provides good control for the set point changes and sudden disturbances. The comparison of the different controllers shows the proposed controller output settles with reference temperature very quickly and it eliminates the overshoot problem

\section{DISCUSSION}

In this study, neuro fuzzy controller for its unique characteristics emerges as a replacement for the previous existing controllers. The merits of the neuro fuzzy controller can be observed as following. The neuro fuzzy controller has improved control quality. The aim of control of heated barrel is to bring the set points during startup as soon as possible while avoiding large overshoots in order to maintain it at current temperature set value. The Table 1 gives various timing specification for the four controllers. From the analysis of the neuro fuzzy controller on the basis of delay time it gives efficient output differences 8.5 times to that of PID controller, 6.35 times than that of neural controllers and 1.5times to that of Fuzzy. Consequently the neuro fuzzy controller produces an output which is 5 times ahead of that of PID, 3 times efficient than neural controller and 1.6 times than that of fuzzy in the rise time analysis. The peak time results states that neuro fuzzy output 6.66 times efficient than PID, 2 times than neural network and 1.66 times than that of Fuzzy controller. With consideration over the settling time the neuro fuzzy controller is 1.15 times efficient than PID, 1.10 times efficient than neural controller and 1.09 times than that of Fuzzy. The set points tracking and disturbance rejection is obtained in the proposed method. 
J. Computer Sci., 7 (5): 671-677, 2011

Table 1: Timing specification of PID, Neural, FLC and Neuro Fuzzy Controller

\begin{tabular}{lllll}
\hline Timing specifications & PID controller & Neural network controller & Fuzzy logic controller & Neuro fuzzy controller \\
\hline Delay time $\left(\mathrm{T}_{\mathrm{d}}\right)$ & $170 \mathrm{Sec}$ & $127 \mathrm{Sec}$ & $25 \mathrm{Sec}$ & $20 \mathrm{Sec}$ \\
Rise time $\left(\mathrm{T}_{\mathrm{r}}\right)$ & $250 \mathrm{Sec}$ & $150 \mathrm{Sec}$ & $80 \mathrm{Sec}$ & $50 \mathrm{Sec}$ \\
Peak time $\left(\mathrm{T}_{\mathrm{p}}\right)$ & $400 \mathrm{Sec}$ & $120 \mathrm{Sec}$ & $100 \mathrm{Sec}$ & $60 \mathrm{Sec}$ \\
Settling time $\left(\mathrm{T}_{\mathrm{s}}\right)$ & $1900 \mathrm{Sec}$ & $1820 \mathrm{Sec}$ & $1800 \mathrm{Sec}$ & $1650 \mathrm{Sec}$ \\
Peak overshoot $(\%)$ & $21 \%$ & 0 & 0 & 0 \\
\hline
\end{tabular}

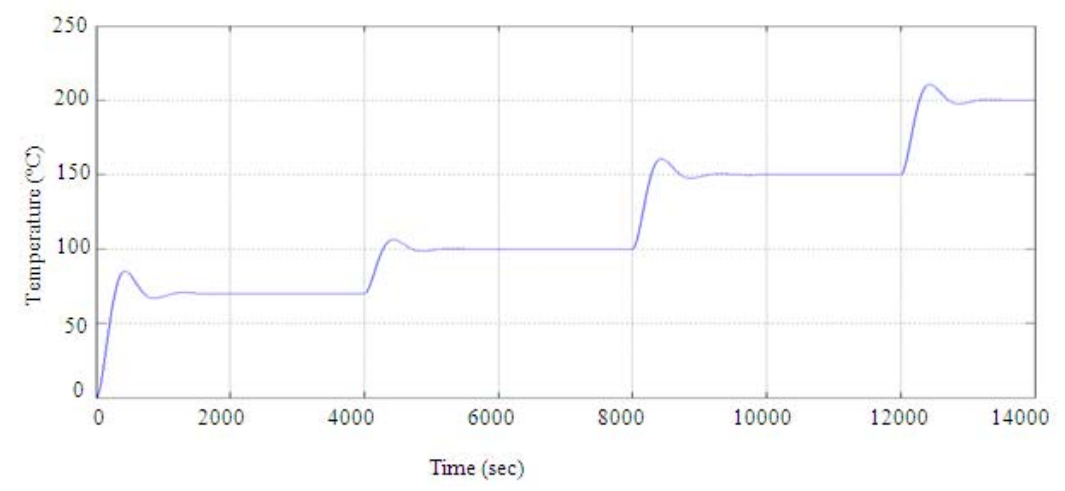

Fig. 7: PID control simulated output at different temperature set points

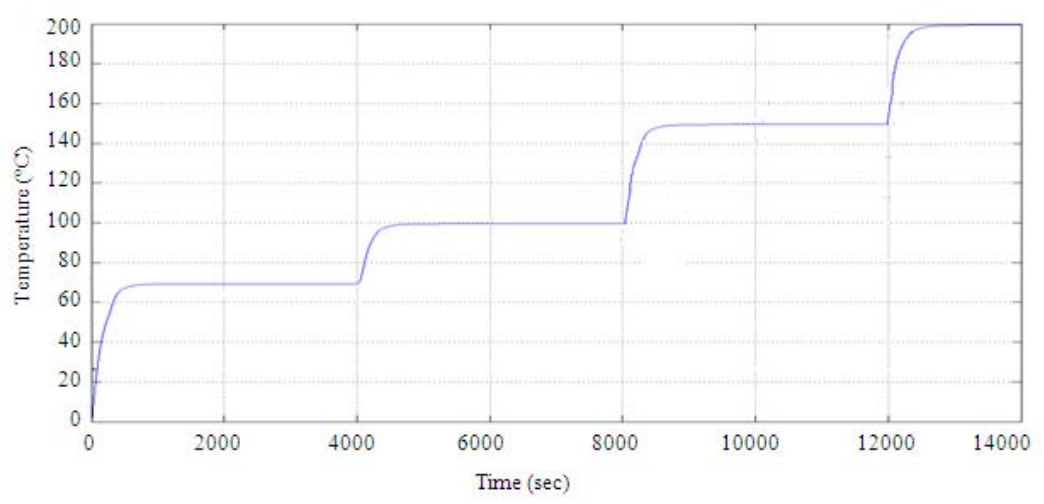

Fig. 8: Neural network simulated output at different temperature set points

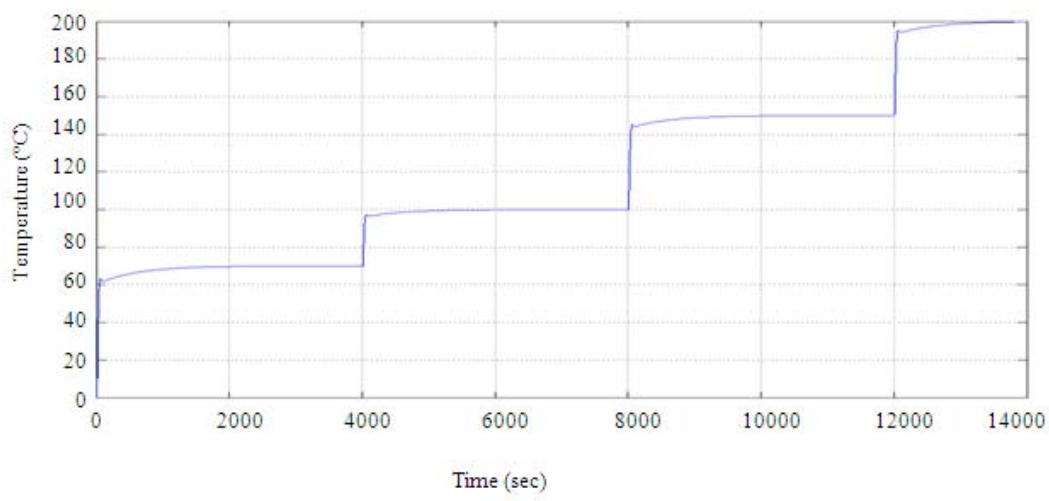

Fig. 9: Fuzzy control simulated output at different temperature set points 


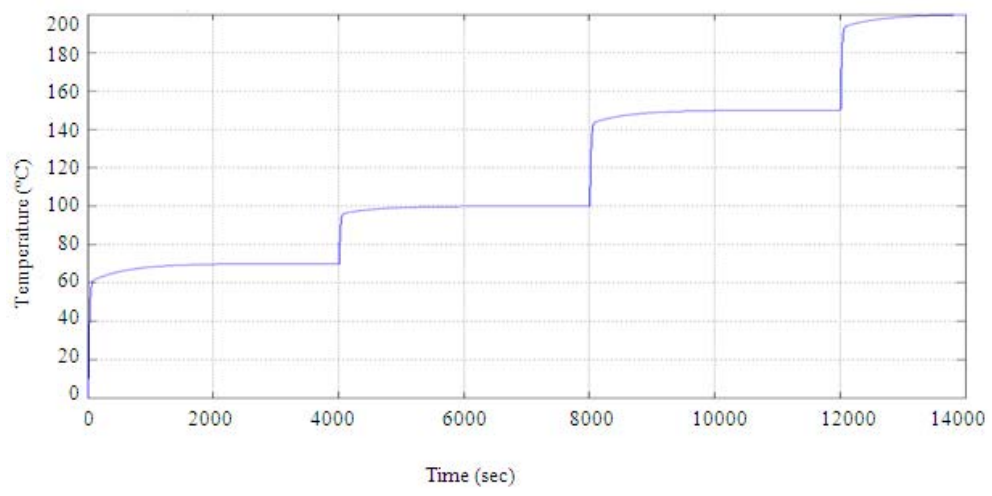

Fig.10: Neuro fuzzy controller simulated output at different temperature set points

\section{CONCLUSION}

The proposed neuro fuzzy controller eliminates sudden input disturbance and maintain the set point temperature in the plastic extrusion system. The simulation results shows neuro fuzzy controller reduces the timing specifications of Fuzzy, Neural and PID controllers. This study demonstrates the effectiveness of intelligent controller on non linear system particular for temperature control in plastic extrusion system. The comparison of performance of the three controllers reveals that the neuro fuzzy controller is superior to the other controllers. From the results the proposed controller is good for set point changes and for stability. With the aid of the supervisory technique, the proposed controller identifies the process variations quickly and provides good controller performance for the set point changes and sudden disturbances. Therefore neuro fuzzy controller will prove especially efficacious in the case of plastic extrusion temperature control system.

\section{REFERENCES}

Ahmad, M.A. and Z. Mohamed, 2009. Hybrid fuzzy logic control with input shaping for input tracking and sway suppression of a gantry crane system. Am. J. Eng. Applied Sci., 2: 241-251. DOI: 10.3844/ajeassp.2009.241.251

Chamsai, T. and P. Jirawattana and Thana Radpukdee, 2010. Sliding mode control with pid tuning technique: An application to a de servo motor position tracking control. Energy Res. J., 1: 55-61. DOI: 10.3844 /erjsp.2010.55.61

Iranian, M.E. and A. Zabihinejad, 2011. Modeling and implementation of generator and network simulator for static exciters usilng MATLAB and labview. J. Applied Sci., 11: 414-425. DOI: 10.3923/jas.2011.414.425
Liang, G.J., Z.H. Xia and Z. Jin, 2010. Fuzzy logic based current control schemes for vector-controlled asynchronous motor drives. Inform. Technol. J., 9: 1495-1499. DOI: 10.3923/itj.2010.1495.1499

Magaji, N. and M.W.Mustafa, 2009. Optimal thyristor control series capacitor neuro-controller for damping oscillations. J. Comput. Sci., 5: 980-987. DOI: $10.3844 /$ jessp. 2009.980 .987

Malar, R.S.M.N. and T. Thyagarajan, 2009. Artificial neural networks based modeling and control of continuous stirred tank reactor. Am. J., Eng. Applied Sci., 2: 229-235. DOI: 10.3844/ajeassp.2009.229.235

Mjalli, F.S., Y.K. Ho and H.K. Yeoh, 2010. Multivariable adaptive predictive model based control of a biodiesel transesterification reactor. J. Applied Sci., 10: 1019-1027. DOI: 10.3923/jas.2010.1019.1027

Oreste, P., 2009.The convergence-confinement method: Roles and limits in modern geomechanical tunnel design. Am. J. Applied Sci., 6: 757-771. DOI: 10.3844/ajassp.2009.757.771

Pratumsuwan, P., S. Thongchai and S. Tansriwong, 2010. A hybrid of fuzzy and proportional-integralderivative controller for electro-hydraulic position servo system. Energy Res. J., 1: 62-67. DOI: 10.3844/erjsp.2010.62.67

Sharifi, D., D. Kazemi and H. Latifi, 2009. Evaluation of tensile strength of the superficial digital flexor tendon in horses subjected to transcutaneous electrical neural stimulation therapeutic regimen. Am. J. Applied Sci., 6: 816-819. DOI: 10.3844/ajassp.2009.816.819

Yuwono, R. Y., 2009. X10 protocol man machine interface implementation using Labview. J. Applied Sci., 9:3562-3568. DOI: 10.3923/jas.2009.3562.3568 